\title{
ORTHODONTIC TOOTH MOVEMENT AND ITS EFFECT ON ORAL TISSUES: A REVIEW
}

\author{
Dr. Anil Singh ${ }^{1}$ \\ 1. Professor \& HOD, Dept of Oral Pathology, Sardar Patel Post Graduate Institute of Dental \& Medical Sciences, Lucknow, India
}

Correspondece : dranilsingh@yahoo.com

\section{INTRODUCTION:}

The success of orthodontic treatment is influenced by a number of factors, including periodontal health, oral hygiene, and orthodontic forces. The development of new methods to accelerate orthodontic tooth movement (OTM) has been sought by clinicians as a way to shorten treatment time, reduce adverse effects such as pain, discomfort, dental caries and periodontal diseases, and minimize iatrogenic damages such as root resorption and the subsequent development of non-vital teeth. ${ }^{1}$

OTM differs markedly from physiologic tooth eruption. Physiological tooth movement is a slow process that occurs mainly in the buccal direction into cancellous bone or because of growth into cortical bone whereas, OTM is uniquely characterized by the abrupt creation of compression and tension regions in the periodontal ligament $(\mathrm{PDL}){ }^{2} \mathrm{~A}$ daunting array of coordinated biochemical reactions occur in and around cells, leading to end points of protein synthesis, mitosis (cell division), and cell differentiation. Mechanically induced, cellmediated time and space changes in bone and soft tissue return the craniomandibular system to homeostasis. ${ }^{3}$

Orthodontic theory involves the remodelling of bone and requires a thorough understanding of bone biology, particularly the relationship between mechanical stress and various types of cells in bone. However, tooth movement is a complicated process that requires changes in periodontal ligament (PDL) as well as in supporting alveolar bone, tissues with different cell populations and remodelling characteristics. ${ }^{4}$

\section{THEORIES OF ORTHODONTIC TOOTH MOVEMENT (OTM):}

Orthodontic tooth movement can be defined as the result of a biologic response to interference in the physiologic equilibrium of the dentofacial complex by an externally applied force. ${ }^{5}$ The quest of the biological foundation of force induced tooth movement led to the proposal of the various mechanisms of tooth movement. (a) Pressure-tension theory

Several researches were conducted by Sandstedt C (1904), Oppenheim A (1911) and Schwartz AM (1932) on the histology of tooth movement that led them to hypothesize the 'pressure-tension theory'. On the pressure side, the biological events are as follows: disturbance of blood flow in the compressed PDL, cell death in the compressed area of the PDL (hyalinization), resorption of the hyalinized tissue by macrophages, and undermining bone resorption by osteoclasts beside the hyalinized tissue, which ultimately results in tooth movement. On the tension side, blood flow is activated where the PDL is stretched, which promotes osteoblastic activity and osteoid deposition, which later mineralizes. ${ }^{6,7,8}$

(b) Bone bending theory

Baumrind S (1969) proposed this theory which states that orthodontic forces routinely produce alveolar bone deflection and that these strains are accompanied by changes in the PDL. ${ }^{7}$ When a force is exerted upon the teeth to be moved two principal changes take place in the alveolar process. First, a bending of the alveolar process occurs; secondly, absorption of the process in advance of the moving teeth and deposition of bone behind it. These changes vary greatly: according to the age of the patient, in different patients of the same age, in the direction of movement and also in the rapidity of movement. ${ }^{4}$

(c) Fluid flow theory and Piezoelectricity

This is a mechanism by which osteocytes respond to mechanical forces and states that locally evoked strain derived from the displacement of fluid in the canaliculi is very important. When loading occurs, interstitial fluid is squeezed through the thin layer of non-mineralized matrix surrounding the cell 
bodies and cell processes, resulting in local strain at the cell membrane and activation of the affected osteocytes. ${ }^{8}$

\section{CELLS INVOLVED IN OTM:}

Osteoblasts and osteoclasts are two important cells that are involved in OTM. ${ }^{3}$ As such many studies have focused on these cells during OTM. Osteoblast cells are involved in bone formation, which begins 40 to 48 hours after the application of orthodontic forces. Osteoblast differentiation starts with stem cells that originated from the bone marrow that migrated into the blood vessels. Migration of mesenchymal stem cells from blood vessel walls, or mesenchymal stem cell precursor activation and preosteoblast formation, occurs about 10 hours after the application of force. This sequence of cellular activities occurs during the development of the stem cell into osteoblast and osteoclast cells and may be useful in determining potential markers associated with OTM. Osteoclast cells are multinucleated cells which degrade and resorb bone. Osteoclast cells which work together with osteoblast cells in bone remodelling are derived from haematopoietic stem cells. ${ }^{1,3}$

\section{MOLECULES INVOLVED IN OTM:}

\section{(i) Prostaglandin}

Prostaglandins (PGs) are a group of chemical messengers and are derivatives of arachidonic acid. They play an important role in promoting bone resorption by stimulating cells to produce cyclic adenosine monophosphate (cAMP), which is an important chemical messenger for bone resorption. ${ }^{8}$ PGE1 and PGE2 are thought to have essential roles in bone resorption. They have a direct action on osteoclasts in increasing their numbers and their capacity to form a ruffled border and effect bone resorption. $^{7}$

\section{(ii) Cytokines}

Cytokines are extracellular signalling proteins that act on nearby target cells in low concentrations in an autocrine or paracrine fashion in cell-to-cell communications. The cytokines that were found to affect bone metabolism and thereby, OTM, include interleukin 1 (IL-1), interleukin 2 (IL-2), interleukin 3 (IL-3), interleukin 6 (IL-6), interleukin 8 (IL-8), tumor necrosis factor alpha (TNF $\alpha$ ), gamma interferon (IFN $\gamma$ ) and osteoclast differentiation factor (ODF). The most potent among these is IL-1 whose actions include attracting leukocytes and stimulating fibroblasts, endothelial cells, osteoclasts, and osteoblasts to promote bone resorption and inhibit bone formation. Osteoblasts are target cells for IL1 , which in turn conveys messages to osteoclasts to resorb bone. ${ }^{7}$

IL-6 can induce osteoclastic bone resorption through an effect on osteoclastogenesis. IL- 6 has been identified in human gingival tissue and cells. The levels of IL- 6 increase significantly 24 hours after mechanical loading and play an important role in initiating tooth movement. ${ }^{8}$

TNF $\alpha$, receptor activator of nuclear factor kappa-B ligand (RANKL) and its receptor activator of nuclear factor kappa B (RANK) have been identified as the key factors that stimulate osteoclast formation and in OTM. ${ }^{8}$

\section{(iii) Neurotransmitters}

The relationship of nerves to tooth movement has been a matter of considerable research. The force-sensing PDL nerve fibers are either unmyelinated $C$ fibers or small myelinated $A \delta$ fibers. The mechanoreceptors are silent in physiological conditions but contain various neuropeptides such as substance $P$, vasoactive intestinal polypeptide, and calcitonin gene-related peptide (CGRP). These neuropeptides are routinely stored in peripheral and central nerve. OTM affects the number, functional properties, and distribution of both mechanosensitive and nociceptive periodontal nerve fibers. Increased immunoreactivity of substance $P$ has been demonstrated in the PDL in the early phases of tooth movement. This neuropeptide has been shown to cause vasodilatation and increased vascular permeability, contributing to increased local blood flow that accompanies inflammation. ${ }^{7}$

\section{(iv) Growth factors}

Bone contains abundant amount of transforming growth factor $\beta$ (TGF $\beta$ ) which has been shown to enhance osteoclast differentiation in hemopoietic cells stimulated with RANKL and M-CSF. ${ }^{7}$

\section{(v) Colony stimulating factors}

Colony-stimulating factors (CSF) include those related to granulocytes (G-CSF), macrophages (M-CSF), or to both cell types (GM-CSF), and might have particular implication in bone remodeling through osteoclast formation and thereby during tooth movement. ${ }^{7}$

\section{VARIOUS TISSUE RESPONSES TO OTM:}

The tissue reaction following orthodontic tooth movement and its relation to functional influences has been a problem frequently discussed during last fifteen years. ${ }^{9}$ The histological changes which occur when forces are applied to teeth are well documented. Teeth appear to lie in a position of balance between the tongue and lips or cheeks. This zone is not completely neutral since tongue forces are usually slightly greater than the lips or cheeks. Very low forces are capable of moving teeth. Classically, ideal forces in orthodontic tooth movement are those which just overcome capillary blood pressure. In this situation bone resorption is seen on the pressure side and bone deposition on the tension side. Teeth rarely move in this ideal way. Usually force is not applied evenly 
and teeth move by a series of tipping and uprighting movements. ${ }^{10}$

\section{BONE REMODELLING AND TOOTH MOVEMENT:}

Bone resorption is crucial to orthodontic treatment, by removing alveolar bone from the path of the moving dental root. In this cell-mediated process, the appearance of osteoclasts is considered to be the requisite first step. ${ }^{7}$ Osteoclasts are essential for orthodontic tooth movement to occur. The origin of osteoclasts during orthodontic treatment is an important yet poorly understood phenomenon, however it is proposed that they originate from hemopoietic organs. ${ }^{11}$ When teeth are moved with bone, the resorption takes place directly on the wall of the alveolus from the periodontal ligament. If this is the case, the activity of the osteoclasts on the stress surface and the osteoblasts on the strain surface are synchronized as a remodelling cycle. Corresponding to the resorption occurring on the alveolar wall in the direction of force, apposition occurs at a certain distance within the alveolar process or on the external surface of the alveolus. ${ }^{12}$

The bone-resorption cascade involves a series of steps directed toward removing both the mineral and the organic constituents of bone matrix by osteoclasts. After the differentiation of osteoclasts, the unmineralized osteoid layer in the bone surface is removed by the lining osteoblasts. These cells produce various enzymes such as MMPs, collagenases, and gelatinases that help osteoclasts in accessing the underlying, mineralized bone. The next step is osteoclast polarization by attaching itself to specific extracellular bone matrix proteins. This is followed by osteoclast activation by local and systemic factors, and production of hydrogen ions (that dissolve the mineral) and proteolytic enzymes (that degrade the organic matrix) in the hemivacuole (localized environment) under the ruffled border of the cell. Osteoclasts ultimately undergo apoptosis, characterized by nuclear and cytoplasmic condensation, and fragmentation of nuclear DNA into nucleosomal sized units. ${ }^{7}$

\section{PDL REMODELLING DURING OTM:}

The most dramatic remodelling changes incident to OTM occur in the PDL. It is likely that PDL cells stimulated by various forces produce local factors that participate in the maintenance, remodelling of the ligament itself and metabolism of adjacent alveolar bone. ${ }^{8}$

In OTM, the remodelling process in periodontal tissues is repeated, and biomechanical properties may be affected by anatomical alterations in the PDL space and alveolar bone height. ${ }^{13}$ Widening of the PDL is observed in tension sites; the tooth has been drawn away from the alveolar bone. In the stretched PDL, several cellular processes are apparently activated, along with an increase in the number of connective tissue cells. This initial phase is followed by deposition of osteoid tissue at the edge of the socket wall. The blood vessels in the PDL tension site become distended, and fibroblasts are rearranged in the direction of strain. The stretched fibroblasts appear spindle shaped in the middle of the PDL and spherical near the alveolar bone. ${ }^{7}$ PDL cells, under mechanical stress, may induce secretion of osteoclasts through upregulation of RANKL expression via PGE2 synthesis during OTM. The number and distribution of patterns of RANKL and RANK expressing osteoclasts change when excessive orthodontic force is applied to periodontal tissue and IL-1 $\beta$ and TNF $\alpha$ are expressed in osteoclasts. ${ }^{8}$ Tissue changes in the compressed PDL are characterized by edema, gradual obliteration of blood vessels and breakdown of the walls of veins, followed by leakage of blood constituents into the extravascular space. Changes seen in fibroblasts at these sites are moderate swelling of the endoplasmic reticulum, formation of vacuoles, and rupture and loss of cytoplasm. This disintegration leaves isolated nuclei, which undergo lysis over a period of several weeks. As long as the ground substance remains in the PDL, the tissue has a glossy appearance. The degenerative process is maintained as long as the pressure persists. With time, accumulated erythrocyte breakdown products in pressure regions might undergo crystallization. ${ }^{7}$ During orthodontic force application, the periodontal ligament (PDL) undergoes hyalinization, a light-microscope term describing the loss of cells from an area of the ligament because of necrosis. This tissue damage prevents the tooth from moving until the adjacent bone and the necrotic tissue are removed by osteoclasts. ${ }^{14}$

It is clear that, as long as ankylosis does not occur, the general trend after force application is preservation of the width of the PDL, a remarkable process involving precisely controlled osteogenic resorption and deposition at specific sites in the paradental tissues. Conclusions from recent studies showed that the PDL might be both the medium of force transfer and the means by which alveolar bone remodels in response to applied forces. ${ }^{7}$

\section{RESPONSE OF GINGIVAL TISSUE TO TOOTH MOVEMENT:}

Gingival fibroblasts are essential for maintaining oral homeostasis. They participate in tissue repair and contribute to tissue remodelling following the application of physiological forces such as mastication. ${ }^{15}$ Two disparate processes occur in the gingiva after the transduction of the orthodontic force. First, there is an injury of the connective tissue manifested by torn and ripped collagen fibers. Second, the genes of both collagen and elastin are activated whereas that of tissue collagenase is inhibited, thus, affecting the extra cellular matrix (ECM) of the gingiva. Therefore, the relapse is probably associated with increase in elasticity of the gingiva that is being retracted and compressed in direction of the tooth movement. ${ }^{16}$

Gross clinical observation of the gingiva during orthodontic treatment showed tissue accumulation and enlargement of gingival papillae when extraction spaces are being closed. This tissue accumulation is attributed to both retraction and compression. Adjacent to this tissue accumulation, vertical clefts of both epithelium 
and connective tissue, which persisted for years after treatment, have been reported. Histologic studies showed discontinuation of transseptal fibers at extraction sites and their reestablishment during the healing phase. These newly formed collagen fibers are coiled and compressed, and have a football-shaped appearance after orthodontic approximation of teeth at extraction sites. There are increased in the amount of oxytalan fibers and glycosamino-glycans (GAGs) in the transseptal fiber area. Data suggests that orthodontic force effects on the gingiva are similar in cases of extraction space closure and rotation corrections. Furthermore, these findings indicate that the cause of relapse after treatment is most likely the increased elasticity of the compressed gingiva, brought about by the biosynthesis of new elastic fibers and GAGs. In this fashion, the gingiva undergoes elastic deformation during orthodontic treatment, capable of causing relapse during the post-treatment retention period. ${ }^{7}$

\section{EFFECT ON TOOTH ROOT AND PULPAL TISSUE:}

The ability to move teeth through bone is dependent on bone being resorbed and tooth roots remaining intact. It is highly probable that all teeth which have undergone orthodontic tooth movement exhibit some degree of microscopic root resorption. Excessive root resorption is found in $3-5 \%$ of orthodontic patients. Some teeth are more susceptible than others; upper lateral incisors can, on an average, lose $2 \mathrm{~mm}$ of root length during a course of fixed orthodontic treatment. There are specific features of appliances which can increase the risk of root resorption. ${ }^{10}$ OTM can directly cause irreversible resorption of the root. ${ }^{17}$ It is an undesirable and the least predictable sequelae of orthodontic treatment. Apical root resorption observed mid- or post-treatment is occasionally of passing interest to the clinician, but usually of little importance raising questions about the longevity of the treated dentition and the stability of the treatment results. Along with other factors, orthodontic forces can initiate and sustain the resorptive process. ${ }^{7,17}$ Whatever the predisposing or influencing factors; most authors believe that there will be no apparent increase in resorption after termination of active orthodontic treatment. Some repair occurs, including smoothing and remodeling of cemental surfaces and the return of the PDL width to normal. Original root contours and lengths are never re-established, but the function of the tooth apparatus is usually not severely affected by the loss of root length. ${ }^{7}$

Various dental procedures, including orthodontic tooth movement, have been reported to adversely affect the dental pulp. In recent years, the alterations in pulpal vasculature and blood flow in response to orthodontic force have gained much attention. Orthodontic forces cause cell damage, inflammatory changes and circulatory disturbances in dental pulp. Calcitonin gene related peptide (CGRP) and substance $P$ present on dental pulp have been associated with the mediation of pulpal inflammation. The progression of pulpal inflammation is also mediated by the production of various cytokines like IL-1, IL-3, IL-6 and TNF $\alpha{ }^{7,8}$

\section{GINGIVAL CREVICULAR FLUID (GCF) AND ORTHODONTIC TOOTH MOVEMENT:}

GCF is an osmotically mediated inflammatory exudate found in the gingival sulcus where it tends to increase in volume with inflammation and capillary permeability. During the course of orthodontic treatment, the force exerted produce a distortion of the PDL extracellular matrix, resulting in cellular shape and cytoskeletal configuration. Such events lead to the synthesis and presence in the deeper periodontal tissues of extracellular matrix components, tissue-degrading enzymes, acids and inflammatory mediators; induce cellular proliferation and differentiation and promote wound healing and tissue remodelling. They produce an alteration in the GCF flow rate and its components. Glycosaminoglycans (GAGs) particularly hyaluronic acid and chondroitin sulphate are believed to be increased in the GCF following OTM. Certain molecules that are found to be increased in the GCF due to orthodontic forces are IL-1 $\beta$, IL-6, TNF $\alpha$, Cathepsin B, RANKL while alkaline phosphatase and leptin (polypeptide hormone) are found to be decreased. , $, 7,8^{2}$

\section{CONCLUSION:}

The studies regarding tissue reaction to orthodontic forces were initiated with the purpose of finding an explanation of the controversies in the literature. The orthodontic displacement of a tooth is the result of a mechanical stimulus, generated by a force applied to the crown of a tooth, which results in acute inflammatory response in periodontal tissues, which in turn may trigger the cascade of biological events associated with bone remodelling. It is evident that, at different stages of tooth movement, different combinations of cell-to-cell and cellto-matrix interactions occur; these determine the nature of the remodelling changes. However, well designed experimental studies are needed for the same in order to evaluate their clinical efficiency and validate their use, as this is an era of evidence based dentistry. 


\section{REFERENCES:}

1. Ariffin SHZ, Yamamoto Z, Abidin IZZ, Wahab RMA, Ariffin ZZ. Cellular and Molecular Changes in Orthodontic Tooth Movement. The Scientific World Journal, 2011; 11: 1788-1803.

2. Meeran NA. The role of gingival crevicular fluid in orthodontic tooth movement - a review. SRM University Journal of Dental Sciences, 2011; 2(2); 129-133.

3. Masella RS, Meister M. Cellular concepts in the biology of orthodontic tooth movement. Am J Ortho Dentofacial Orthop (2006), 129: 458-468.

4. Meikle MC. The tissue, cellular and molecular regulation of orthodontic tooth movement: 100 years after Carl Sandstedt. Eur J Orthod, 2006; 221-240.

5. Proffit WR. Biologic basis of orthodontic therapy. In: Proffit WR, Fields HW, editors. Contemporary Orthodontics. 3rd ed. St Louis: Mosby; 2000.

6. von-Bohl M, Jagtman AMK. Hyalinization during orthodontic tooth movement: a systematic review. Eur J Ortho, 2009; 30-36.

7. Krishnan V, Davidovitch Z. Cellular, molecular and tissue level reaction to orthodontic forces. Am J Orthod Dentofacial Orthop, 2006; 129: 469-460.

8. Meeran NA. Biological response at the cellular level within the periodontal ligament on application of orthodontic force- an update. J Ortho Sci, 2012; 1(1): 2-10.

9. Reitan K. The tissue reaction as related to the functional factor. Eur J Orthod, 2007; 58-64.

10. Harry DR, Sandy J.Orthodontis Part II: Orthodontic tooth movement. Br Dent J, 2004; 196(7): 391-394.

11. Tsay TS, Chen M, Oyen JO. Osteoclast activation and recruitment after application of orthodontic force. Am J Orthod Dentofacial Orthop, 1999; 115: 323-30.

12. Melsen B. Biological reaction of alveolar bone to orthodonotic tooth movement. Angle Orthod, 1999; 69(2): 151-158.

13. Tanne K, Inoue Y, Sakuda M. Biochemical behaviour of the periodontium before and after orthodontic tooth movement. Angle Orthod, 1995; 65(2): 123-128.

14. Rody WJ, King GJ, Gu G. Osteoclast recruitment to sites of compression in orthodontic tooth movement. Am J Orthod Dentofacial Orthop, 2001; 120: 477-89.

15. Danciu TE, Gagari E, Adam RM, Damoulis PD, Freeman MR. Mechanical Strain Delivers Anti-apoptotic and Proliferative Signals to Gingival Fibroblasts. J Dent Res, 2004; 83: 596-601.

16. Redlich M, Shoshan S, Palmon A. Gingival response to orthodontic force. Am J Orthod Dentofacial Orthop, 1999; 116: $152-158$.

17. Sameshima GT, Sinclair PM. Predicting and preventing root resorption- Part 1 Diagnostic factor. Am J Orthod Dentofacial Orthop, 2001; 119: 505-510. 